\title{
Research
}

\section{The value of comparing health outcomes in cohort studies: An example of self-rated health in seven studies including 79653 participants}

Kaarin J Anstey, Peter Butterworth, Tim D Windsor, Richard Burns, Kerry Sargent-Cox, Chwee von Sanden and Helen Christensen

Centre for Mental Health Research, Australian National University, Canberra, Australian Capital Territory, Australia

\section{Heather Booth}

Research School of Social Sciences, Australian National University, Canberra, Australian Capital Territory, Australia

\section{Leon A Simons}

St Vincent's Clinical School, University of New South Wales, Sydney, New South Wales, Australia

\section{Julie E Byles and Richard Gibson}

Research Centre for Gender, Health and Ageing, University of Newcastle, Newcastle, New South Wales, Australia

\section{Mary A LuszCZ}

School of Psychology, Flinders University, Adelaide, South Australia, Australia

\section{Jonathan E Shaw}

International Diabetes Institute, Melbourne, Victoria, Australia

\section{Gerald A Broe}

Prince of Wales Clinical School, University of New South Wales, Sydney, New South Wales, Australia

\section{Colette Browning}

Monash Institute of Health Services Research, Monash University, Melbourne, Victoria Australia

\section{Paul Mitchell}

Department of Ophthalmology, University of Sydney, Sydney, New South Wales, Australia

\section{Robert G Cumming}

School of Public Health, University of Sydney, Sydney, New South Wales, Australia

\section{Hal Kendig}

College of Health Sciences, University of Sydney, Sydney, New South Wales, Australia

Objective: To demonstrate the value of comparing data from multiple cohort studies using the example of self-rated health (SRH).

Methods: Seven Australian cohort studies including comparable data on SRH were identified. Comparisons of the

Correspondence to: Associate Professor Kaarin J Anstey, Ageing Research Unit, Centre for Mental Health Research, Australian National University. Email: Kaarin.anstey@anu.edu.au distributions of SRH were conducted, and logistic regression was used to evaluate age, sex and education effects within studies. A nationally representative survey was used as a statistical reference to determine how studies differed from the Australian population in frequencies of responses.

Results: Ratings of SRH declined with increasing age. Low education was associated with higher frequencies of fair/poor SRH even in young adulthood but there were no sex differences. Results for smaller studies did not necessarily differ from nationally representative studies.

Conclusion: Collaborative reanalysis of Australian cohort permits analysis of health outcomes from a large numbers of participants. Health outcomes and their sociodemographic determinants may be more comprehensively evaluated through such collaborative projects.

Key words: cohort studies, education, epidemiology, health status indicators, sex differences.

\section{Introduction}

There is now a large amount of data collected on ageing from Australian studies, providing potential for collaborative reanalysis projects that will greatly advance our knowledge of ageing in Australia. To date, there have been some examples of pooling data from two or more studies [1], but the potential to be gained from collaborative reanalysis remains relatively unexplored in the Australian context. The present paper reports the results of a demonstration project that investigated self-rated health (SRH) in seven Australian longitudinal studies, to illustrate the value of bringing these separate data sources together and to document some of the factors that need to be considered when conducting this type of research.

The outcome variable of interest for this collaborative project was the widely used SRH question with a general reference point, that is, the item asks individuals to rate their health in general, as opposed to asking them to rate their health in relation to their peers, or their previous health. This item is included in the SF-36 health measure [2] that has been used in several large national Australian surveys as well as smaller cohort studies and is also used as an individual item in some studies. SRH is a key indicator of well-being, and is used in research studies that span a range of disciplines including economics, psychology, medicine, demography, sociology and public health. SRH tends to be associated with most health outcomes in late life. For example, a recent meta-analysis of 22 cohort studies found that those reporting 'poor' on a general measure of SRH had twice the risk of mortality than those 
reporting their health as 'excellent' [3]. In very old adults poor SRH has been found to be associated with increased risk of depression [4] and driving cessation [5]. Poor or fair SRH has been associated with higher levels of psychological distress in young, midlife and older adults in the PATH Through Life Project [6]. In addition, SRH has been found to be a strong independent predictor of stroke [7], functional decline [8], nursing home entry [9], and increased health service utilisation $[10,11]$.

These associations make SRH a key variable for policy makers to identify groups at risk of poor health outcomes. It provides a simple global indicator of well-being that may be used to analyse health differentials based on age, sex, geographical region and education level [12].

\section{Aims}

The specific aims addressed by this demonstration project were: (i) to determine the feasibility of working with data from seven cohorts; (ii) to determine the distribution of 'excellent or very good', 'good' and 'fair or poor' SRH over the adult lifespan in Australian adults; (iii) to describe how SRH differs between sex and education groups; and (iv) to evaluate how studies conducted within a state, city or territory compare with a nationally representative sample on the distribution of SRH.

\section{Methods}

\section{Studies included in the analyses}

Through a search of the Ageing Well Data Archive located at the Australian Social Science Data Archive, personal contact with study investigators and a review of a recent publication describing longitudinal studies of ageing in Australia, we identified Australian longitudinal studies that had comparable items measuring SRH [13]. Ten potential studies contained SRH data, of which seven included compatible measures of SRH. These included the Australian Longitudinal Study of Ageing (ALSA) [14], the Australian Diabetes, Obesity and Lifestyle Study (AusDiab) [15], the Household, Income and Labour Dynamics in Australia (HILDA) Survey; data drawn from the confidentialised unit record file (Release 4.1) [16], the Australian Longitudinal Study of Women's Health (ALSWH) [17], the Canberra Longitudinal Study of Ageing (CLS) [18], the Personality And Total Health Through Life Project (PATH) [19] and the Dubbo Study (Dubbo) [20]. Characteristics of the studies, including year of commencement, sampling frame, age range, sample size and domains of interest, are reported in Table 1. HILDA data for this analysis were restricted to those participants aged 20 years or older. The SRH item was included in the HILDA Survey in both a personal interview and as an item in the SF-36 which was included in a selfcompletion questionnaire. Data from the face-to-face interview were used in this study to maximise the available data. Only data from the first wave of each study were included.

Three further studies, the Sydney Older Person Study [21] $(n=647$; age range 49-97); the Blue Mountains Eye Study [22] ( $n=3654$; age range 65+) and the Melbourne Longitudinal Studies on Healthy Ageing [23] ( $n=1000$; age range $75+)$, included measures of SRH that did not have compatible reference points with the other studies. These are considered briefly to illustrate the problems of comparing items that appear similar but which differ in terms of reference point.

\section{Measures}

\section{Self-rated bealth}

The SRH item that was considered compatible across the seven included studies had a general reference point, meaning that the question was not referenced to age or time but asked about health 'in general' (ALSA, HILDA, PATH, AusDiab, ALSWH and Dubbo) or 'overall' (CLS).

Six studies had the following five-item response scale to the SRH question: 1 = excellent; 2 = very good; 3 = good; 4 = fair; $5=$ poor, while CLS used a four-item response scale: 1 = excellent; 2 =good; $3=$ fair; $4=$ poor. To harmonise the SRH variable, the following coding was used: $1=$ fair/poor; 2 = good; $3=$ excellent/very good. For logistic regression, these

\section{Table 1: Study characteristics at baseline assessment}

\begin{tabular}{|c|c|c|c|c|c|}
\hline Study, year & Sampling frame & Age & $n$ & Participant rate & Domains studied \\
\hline ALSA, 1992 & South Australian electoral roll & $65+$ & 2087 & $53 \%$ & $\begin{array}{l}\text { Physical and mental health, sensory function, } \\
\text { cognition, well-being, social networks and support }\end{array}$ \\
\hline ALSWH, 1996 & $\begin{array}{l}\text { Medicare database, over-sampled rural } \\
\text { and remote }\end{array}$ & $\begin{array}{l}18-23 \\
45-50 \\
70-75\end{array}$ & $\begin{array}{l}14739 \\
14011 \\
12762\end{array}$ & $\begin{array}{l}41 \% \\
54 \% \\
38 \%\end{array}$ & $\begin{array}{l}\text { Health, service utilisation, life stages and key } \\
\text { events, work and leisure activity, exercise, lifestyle }\end{array}$ \\
\hline CLS, 1990 & $\begin{array}{l}\text { Canberra and Queanbeyan electoral rolls, oversampled } \\
\text { older age groups and nursing home residents }\end{array}$ & $70+$ & 1000 & $69 \%$ & $\begin{array}{l}\text { Mental health, physical health, activity, memory } \\
\text { and cognition }\end{array}$ \\
\hline HILDA, 2001 & Australian households & $15+$ & 13969 & $66 \%$ & $\begin{array}{l}\text { Economic and subjective well-being, labour market } \\
\text { dynamics, family structure }\end{array}$ \\
\hline PATH, 1999 & Canberra and Queanbeyan electoral rolls & $\begin{array}{l}20-25 \\
40-45 \\
60-65\end{array}$ & $\begin{array}{l}2404 \\
2528 \\
2551\end{array}$ & $\begin{array}{l}19 \% \\
28 \% \\
53 \%\end{array}$ & $\begin{array}{l}\text { Mental and physical health, substance use, } \\
\text { cognition, genetics, psychosocial characteristics }\end{array}$ \\
\hline AusDiab, 1999 & Australian households & $25+$ & 11247 & $82 \%$ & Diabetes, obesity, lifestyle \\
\hline Dubbo, 1988 & Community-dwelling Dubbo residents & $59-98$ & 2085 & $73 \%$ & $\begin{array}{l}\text { Physical health and mortality, health service } \\
\text { utilisation, dementia, social engagement, retirement }\end{array}$ \\
\hline
\end{tabular}

ALSA, Australian Longitudinal Study of Ageing; ALSWH, Australian Longitudinal Study of Women's Health; AusDiab, Australian Diabetes, Obesity and Lifestyle Study; CLS, Canberra Longitudinal Study of Ageing; HILDA, Household, Income and Labour Dynamics in Australia; PATH, Personality and Total Health Through Life Project. 
codes were further collapsed into two categories $(1=$ fair/poor vs $0=$ excellent/very good/good).

\section{Age and sex}

Five studies involved single cohorts with relatively wide age-ranges, and two studies involved three narrower cohorts of different ages. PATH consisted of three cohorts with age ranges of 20-26, 40-46 and 60-66. (Although the PATH study aimed to sample ages $20-24,40-44$ and $60-64$ from the electoral roll, a limited number of participants were older than this at the time of interview.) ALSWH consisted of three cohorts with age ranges of $18-23,45-50$ and $70-75$. In both PATH and ALSWH, the three cohorts are referred to as young, middle aged and old. Age was treated continuously in logistic regression analyses and categorically in 5- or 10-year age groups for analyses comparing SRH relative frequencies from separate studies with those from the nationally representative HILDA. In all studies, the categories for sex were male and female (with the exception of ALSWH where all participants were female).

\section{Education (age left school)}

The reporting of information on educational experience differed between studies. A review of alternative questions, distributions of variables, and possible coding schemes was undertaken. A large proportion of study participants had left school after junior high school, at approximately 14 years.

Except for PATH, all studies asked participants, 'How old/ What age were you when you left school?' Three different coding schemes were used. ALSA and AusDiab used a seven-item response scale $(1=$ never went to school; $2=$ under $14 ; 3=14$; $4=15 ; 5=16 ; 6=17 ; 7=18+$ ). ALSWH young and middleaged cohorts used a six-item response scale $(1=$ still at school; $2=$ never attended; $3=14$ years or under; $4=15-16$ years; $5=17-18$ years; $6=19$ years or older). ALSWH old cohort used a five-item response scale $(1=$ never attended; $2=14$ years or under; $3=15-16$ years; $4=17-18$ years; $5=19$ years or older). Participants in the CLS, HILDA and Dubbo studies provided a raw age in answer to the question on age left school.

PATH asked participants, 'What is the highest level of schooling you have completed?' with a five-item response scale ( 1 = some primary; $2=$ all primary; $3=$ some of secondary; $4=$ three/four years of secondary; $5=$ five/six years of secondary).

The education variables in each study were recoded to a common binary item of age at which left school $(0=14$ years old or younger and low levels of education (low education); $1=15$ years old or older and high levels of education (high education)).

\section{Statistical analysis and procedure}

After the analysis of codebooks from the seven studies, researchers from the Centre for Mental Health Research (CMHR) prepared syntax for each study to recode the variables of interest to the same format and coding structure (e.g. male $=1$, female $=0$ ). In some instances, syntax was sent to study owners who then ran the analyses and provided the results for compilation. In other cases, study custodians sent data to CMHR for recoding and analysis. Therefore, the methodology for this demonstration project did not necessarily require sharing of data among study owners.

Percentages of the categories of SRH were plotted across studies, by age, sex and education. Cell sizes with less than 10 observations were excluded from these figures. Logistic regressions (on SRH fair/poor vs all other categories) were used to evaluate the effects of age, education and sex within each study. Low education and male were used as reference categories. In addition, in order to test whether the SRH distributions (fair/poor, good, excellent/very good) for participants aged 60 years and older from each study differ from the Australian population, comparison was made using $\chi^{2}$ tests with HILDA. Although AusDiab would have been a suitable nationally representative reference study, in this instance, HILDA was chosen as a statistical comparison to provide expected frequencies. An alpha level of 0.01 was used.

Analyses of most studies were conducted using SPSS at CMHR, while one study (ALSWH) conducted their own analyses using SAS, after adapting the SPSS syntax provided by CMHR. Analysis of the HILDA Survey data used the svy procedures of STATA 9.2 to derive estimates that account for the complex clustering and stratification of the survey design.

\section{Results}

\section{Excluded studies}

Figure 1 shows the distributions of SRH among the three excluded studies and a comparison study, HILDA. The differences in the distributions of the variable may be the result of the differences in the item, rather than the study characteristics.

\section{Main effects of age, education and sex on self-rated health} Table 2 shows the results of logistic regression analyses undertaken for each study to test for associations of age, education and sex with fair/poor SRH. There was a general finding across studies that increasing age is associated with fair/poor SRH. The small odds ratios for age are the result of the measurement of age by year. For example, using ALSA data, odds ratios increased when age was grouped into 5-year intervals from 1.03 (95\% CI: $1.01-1.04)$ to 1.13 (95\% CI: $1.05-1.22$ ), and to 1.265 (95\% CI: $1.12-1.44)$ when grouped by 10 -year intervals. Sex was not associated with SRH. High education was associated with a reduced likelihood of rating health as fair or poor in all studies except the CLS.

\section{Comparison of observed and expected frequencies of self-rated health responses}

Table 3 shows the frequency of SRH responses by age and sex for HILDA and for each study with $\chi^{2}$ tests of the difference between the frequencies of each study and the expected frequencies based on HILDA. Differences reached statistical significance for all studies except for ALSA and ALSWH. 

with excluded studies.

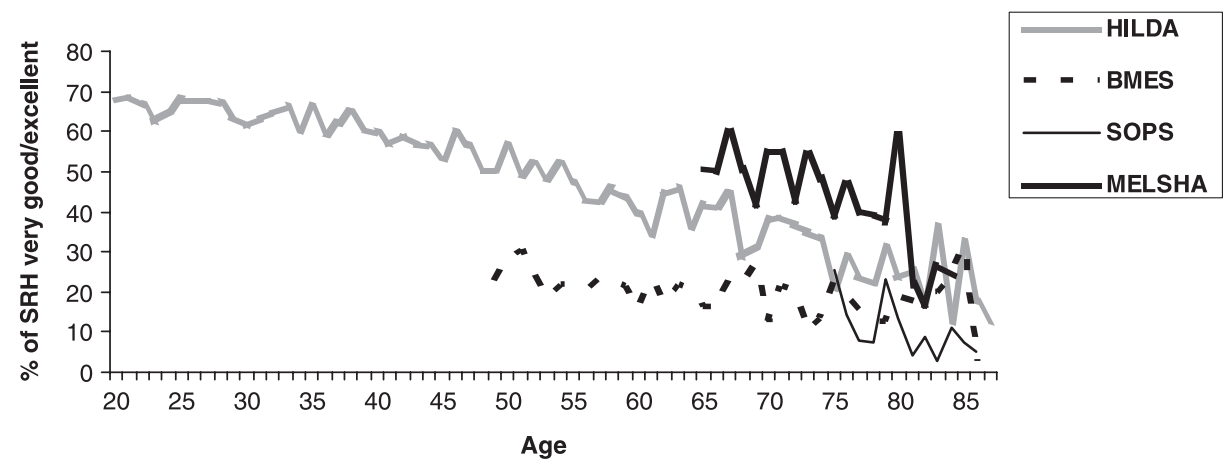

Table 2: Within-study main effects of age, education and sex on self-rated health

\begin{tabular}{|c|c|c|c|}
\hline \multirow[t]{2}{*}{ Study } & \multicolumn{3}{|c|}{ Odds ratio $(95 \%$ Cl) } \\
\hline & Age & Sex (female) & Education (high) \\
\hline ALSA & $1.03(1.01-1.04)^{\star \star}$ & $0.89(0.73-1.07)$ & $0.69(0.57-0.83)^{\star \star \star}$ \\
\hline ALSWH & $1.02(1.01-1.01)^{\star \star \star}$ & NA & $0.51(0.47-0.54)^{\star \star \star}$ \\
\hline CLS & $1.00(1.00-1.00)$ & $1.23(0.93-1.62)$ & $1.00(0.75-1.33)$ \\
\hline HILDA & $1.03(1.03-1.03)^{\star \star \star}$ & $0.92(0.83-1.01)$ & $0.47(0.41-0.54)^{\star \star \star}$ \\
\hline AusDiab & $1.02(1.01-1.02)^{\star * *}$ & $0.87(0.78-0.98)$ & $0.51(0.44-0.58)^{\star \star \star}$ \\
\hline PATH & $1.00(1.00-1.00)$ & $1.02(0.88-1.19)$ & $0.58(0.49-0.68)^{\star \star \star}$ \\
\hline
\end{tabular}

${ }^{\star} P<0.05 ;{ }^{* *} P<0.01 ;{ }^{* \star \star} P<0.001$.

ALSA, Australian Longitudinal Study of Ageing; ALSWH, Australian Longitudinal Study of Women's Health; AusDiab, Australian Diabetes, Obesity and Lifestyle Study; CLS, Canberra Longitudinal

Study of Ageing; HILDA, Household, Income and Labour Dynamics in Australia; NA, not available; PATH, Personality and Total Health Through Life Project.

In PATH, the excellent/very good response category was higher than HILDA, while small differences were found for good, and moderate differences were found for the fair/poor response with a lower frequency of responses in PATH. Significant $\chi^{2}$ statistics were reported for both age groups of CLS participants. The 70-79 age group reported good SRH more frequently than HILDA participants. This was balanced by lower frequencies of both excellent/very good and fair/poor SRH in CLS than in HILDA. Similarly at age 80-89, CLS participants reported good SRH more frequently than expected, with lower frequencies of excellent/very good and fair/poor SRH. However, these differences must be considered in light of the different response scale used in the CLS.

A different pattern of reporting was found for Dubbo study participants of both sexes aged $80-89$. They more frequently reported 'excellent/very good' SRH than HILDA participants of the same age, comparable frequencies for 'good' SRH, and lower frequencies for fair/poor SRH, indicating a general shift of the distribution towards better SRH.

Response frequencies for the female AusDiab participants aged 60-69 and 70-79 paralleled the CLS with higher frequencies of 'good' SRH and lower frequencies of 'excellent/very good' and 'fair/poor' SRH. Male participants aged 80-89 were an exception to this pattern in that they reported a higher frequency of 'excellent/very good' SRH than their HILDA peers.

Figure $2(\mathrm{a}-\mathrm{f})$ shows the distribution of the SRH in all seven studies for males and females by age in years. These show the general trend of declining SRH with age except for a possible improvement in late life suggested by the results from older participants in ALSA. Figure 3 (a-f) shows the distribution of SRH for high and low levels of education in years. These figures demonstrate that low education is associated with poorer SRH even in young adulthood, but that even in those with high levels of education, a decline in age is evident.

\section{Discussion}

The present study documents a basic exercise in the comparative analysis of a key outcome variable across seven Australian Cohort Studies, comprising a total of 79653 participants. The key results demonstrated an unequivocal deterioration in SRH by age until the mid-80s, and suggest a reversal of this pattern in the very old. The study found an absence of sex differences in SRH patterns, and that individuals with low levels of education consistently report poorer SRH, especially in young adulthood. By identifying consistencies in associations across different cohorts and samples, the results also demonstrate the potential value in combining data from multiple sources. From the perspective of Australian gerontologists, a notable observation from this demonstration project is the lack of Australian data on the oldest old.

The finding that the deterioration in SRH appears to be reversed at very old ages merits further examination. Given the association between poor SRH and a greater probability of death, selection effects will occur: those with better SRH will survive longer and come to comprise a larger proportion of the total. From this, it would be expected that the relative frequencies of fair/poor SRH in Figures 2 and 3 would decrease with increasing age. The fact that they do not (at most ages) apparently reflects an increase in the propensity to report fair/poor health as people age, which is stronger than the selection effect. It may be that at very old ages, the selection effect is sufficiently 
Table 3: Frequencies of self-rated health ratings in older adults compared with HILDA

\begin{tabular}{|c|c|c|c|c|c|}
\hline Study by sex and age group & & Excellent/very good $n(\%)$ & Good $n(\%)$ & Poor/fair $n(\%)$ & $\chi_{\mathrm{df}=2}^{2}(P$-value $)$ \\
\hline & Male & & & & \\
\hline & $60-69$ & $321(45)$ & $256(36)$ & $143(20)$ & \\
\hline & $60-66$ & $205(38)$ & $171(33)$ & $145(28)$ & \\
\hline & $65-69$ & $108(39)$ & $93(33)$ & $69(24)$ & \\
\hline & $70-79$ & $150(32)$ & $161(34)$ & $161(34)$ & \\
\hline & $80-89$ & $36(24)$ & $40(27)$ & 72 (49) & \\
\hline \multirow[t]{7}{*}{ HILDA reference age ranges } & Female & & & & \\
\hline & $60-69$ & $147(21)$ & $400(56)$ & $167(23)$ & \\
\hline & $60-66$ & $228(43)$ & $166(32)$ & $133(25)$ & \\
\hline & $65-69$ & 131 (39) & $122(36)$ & 85 (25) & \\
\hline & $70-79$ & $184(33)$ & $184(33)$ & $196(35)$ & \\
\hline & $70-75$ & $128(35)$ & $123(33)$ & $118(32)$ & \\
\hline & $80-89$ & $56(28)$ & 66 (33) & $81(40)$ & \\
\hline \multirow[t]{2}{*}{ PATH 60-66 } & Male & 777 (59) & 378 (29) & $161(12)$ & $19.12(<0.001)$ \\
\hline & Female & $718(58)$ & $354(29)$ & $159(13)$ & $11.50(0.003)$ \\
\hline \multirow[t]{2}{*}{ ALSA 65-69 } & Male & 5 (29) & $4(24)$ & $8(47)$ & Not tested: cells $<10$ \\
\hline & Female & $52(42)$ & 39 (32) & $32(26)$ & $0.88(0.643)$ \\
\hline \multirow[t]{2}{*}{ ALSA 70-79 } & Male & 217 (39) & $175(31)$ & $170(30)$ & $2.27(0.322)$ \\
\hline & Female & $203(39)$ & $180(34)$ & $141(27)$ & $3.01(0.222)$ \\
\hline \multirow[t]{2}{*}{ ALSA 80-89 } & Male & $144(34)$ & $121(29)$ & 159 (37) & $6.46(0.040)$ \\
\hline & Female & $119(36)$ & $90(28)$ & $119(36)$ & $3.86(0.145)$ \\
\hline \multirow[t]{2}{*}{ CLS 70-79 } & Male & 54 (16) & $200(57)$ & 94 (27) & $26.06(<0.001)$ \\
\hline & Female & $63(20)$ & 171 (53) & $88(27)$ & $19.66(<0.001)$ \\
\hline \multirow[t]{2}{*}{ CLS 80-89 } & Male & $28(23)$ & $59(48)$ & $37(30)$ & $23.08(<0.001)$ \\
\hline & Female & 17 (14) & 64 (53) & 40 (33) & $20.58(<0.001)$ \\
\hline \multirow[t]{2}{*}{ Dubbo 60-69 } & Male & $321(45)$ & $256(36)$ & $143(20)$ & $2.76(0.252)$ \\
\hline & Female & $426(52)$ & $242(29)$ & 156 (19) & $5.24(0.073)$ \\
\hline \multirow[t]{2}{*}{ Dubbo 70-79 } & Male & $243(45)$ & $173(32)$ & $130(24)$ & $5.92(0.052)$ \\
\hline & Female & $243(45)$ & 173 (32) & $130(24)$ & $7.80(0.020)$ \\
\hline \multirow[t]{2}{*}{ Dubbo 80-89 } & Male & $42(51)$ & $21(25)$ & 20 (24) & $40.89(<0.001)$ \\
\hline & Female & 80 (49) & 54 (33) & 30 (18) & $28.23(<0.001)$ \\
\hline \multirow[t]{2}{*}{ AusDiab 60-69 } & Male & $303(37)$ & $344(42)$ & $174(21)$ & $2.89(0.236)$ \\
\hline & Female & $325(35)$ & $434(47)$ & $161(18)$ & $9.88(0.007)$ \\
\hline \multirow[t]{2}{*}{ AusDiab 70-79 } & Male & $163(29)$ & $255(46)$ & $138(25)$ & 7.00 (0.030) \\
\hline & Female & $178(26)$ & $319(47)$ & $175(26)$ & $10.09(0.006)$ \\
\hline \multirow[t]{2}{*}{ AusDiab 80-89 } & Male & $57(36)$ & 54 (34) & $46(29)$ & $15.606(<0.001)$ \\
\hline & Female & 37 (22) & $77(46)$ & $55(33)$ & $7.77(0.021)$ \\
\hline ALSWH 68-69 & Female & $4(10)$ & $20(49)$ & $17(41)$ & Not tested: cells $<10$ \\
\hline ALSWH 70-75 & Female & 3941 (33) & 4725 (39) & 3376 (28) & $1.64(0.440)$ \\
\hline
\end{tabular}

ALSA, Australian Longitudinal Study of Ageing; ALSWH, Australian Longitudinal Study of Women's Health; AusDiab, Australian Diabetes, Obesity and Lifestyle Study; CLS, Canberra Longitudinal Study of Ageing; HILDA, Household, Income and Labour Dynamics in Australia; PATH, Personality and Total Health Through Life Project.

strong that it outweighs the increased propensity to report fair/ poor health.

Smaller longitudinal studies drawn from specific geographical areas are often regarded as less than optimal because they are not representative at the national level. However, the results shown here suggest that even two nationally representative studies, such as HILDA and AusDIAB, may show different SRH distributions by age, while smaller studies may not differ from the nationally representative study. ALSA did not differ from HILDA in the distribution of SRH, while the Dubbo study only differed in one age group, despite being a rural sample. It appears that smaller rural and urban studies are not necessarily different from national studies in key outcomes. Therefore, an important conclusion from this study is that we can be optimistic about the inclusion of studies conducted in specific geographical areas, in meta-analyses and projects using pooled data.

The results of the present study should be interpreted in the context of methodological considerations. First, the studies were conducted in different years. The HILDA began in 2001, whereas baseline for other studies ranged from 1990 to 1999 .
Our analyses do not take into account potential cohort effects or the different participation rates among studies. Assumptions were made in the coding of the education variable. Different states in Australia have different education systems, both now and historically, when the older participants in these studies were school aged. In addition, years of education or age left school does not take into account the quality of education received. Nevertheless, consistent effects were found when using the dichotomous variable chosen for this study, suggesting that education has a strong association with SRH, even where its measurement lacks sensitivity.

A further lesson learned from this demonstration project is the difficulty in interpreting results from studies that use different response scales in reference to the same variable. The CLS results tended to be inconsistent with the other studies. Without further investigation of the psychometric properties of SRH items, it is not possible to determine whether these inconsistencies reflect true differences in SRH between samples, or as would appear likely, measurement artefacts. Such issues need to be fully investigated before pooling of variables across studies can be conducted. 
Figure 2: Frequencies of SRH responses in males and females according to age.

(a) SRH fair/poor by age for males

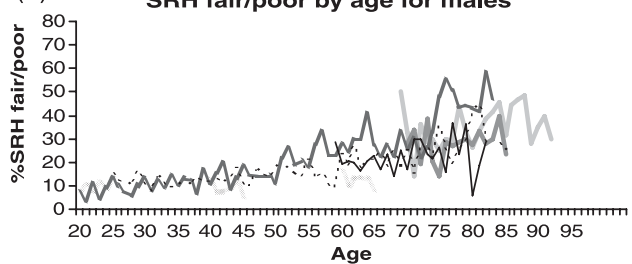

(b)

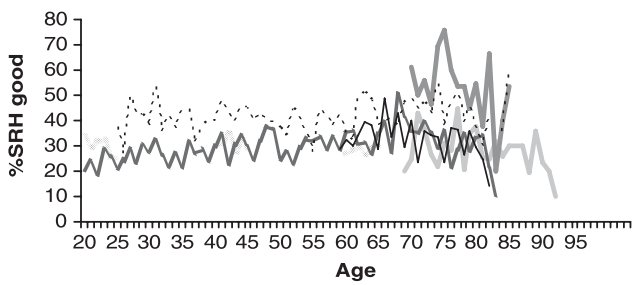

(c) SRH excellent/very good by age for males

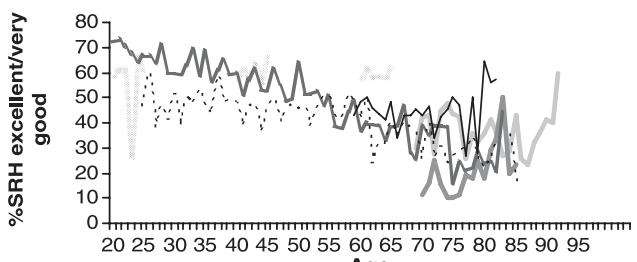

Age (d)

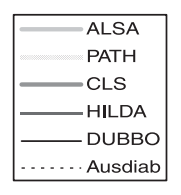

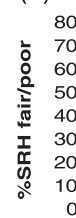
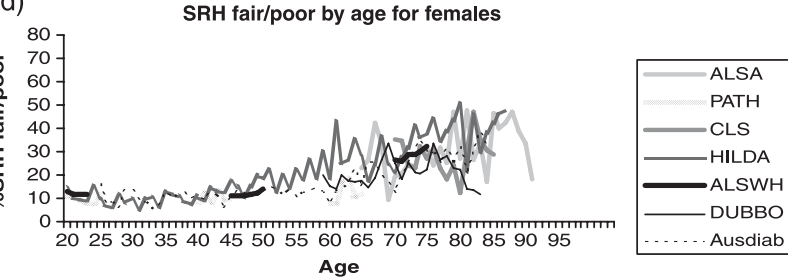

(e)
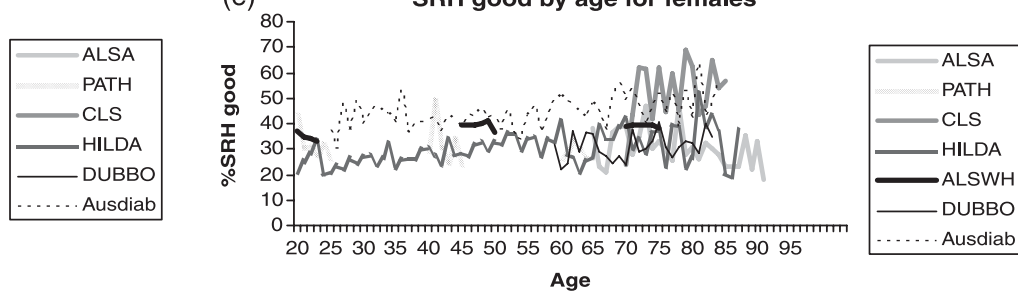

Age

(f) SRH excellent/very good by age for females

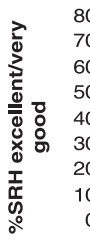

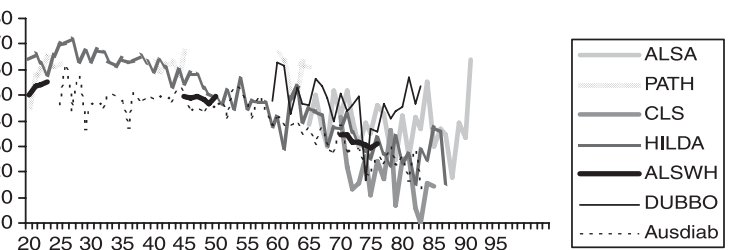

Age

Figure 3: Frequencies of SRH responses in high and low education groups by age.

(a)

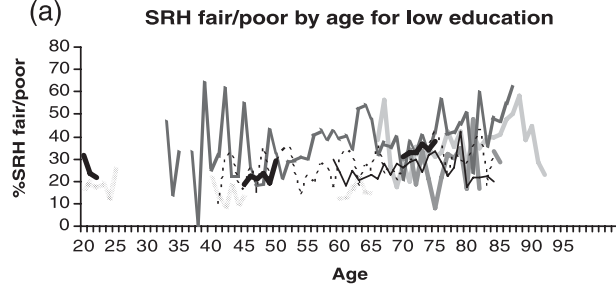

(b) SRH good by age for low education

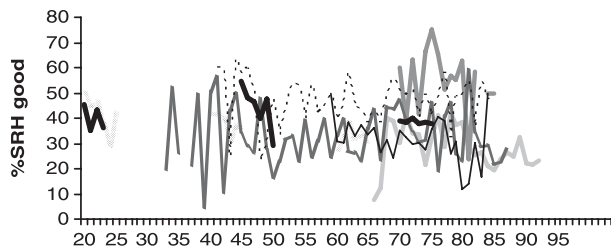

Age

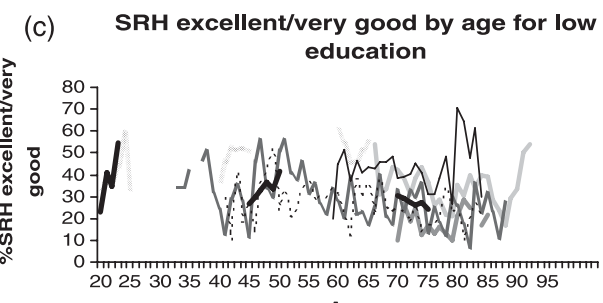

Age (d) SRH fair/poor by age for high education
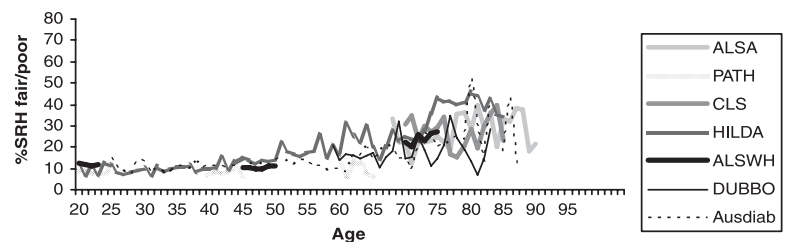

(e)
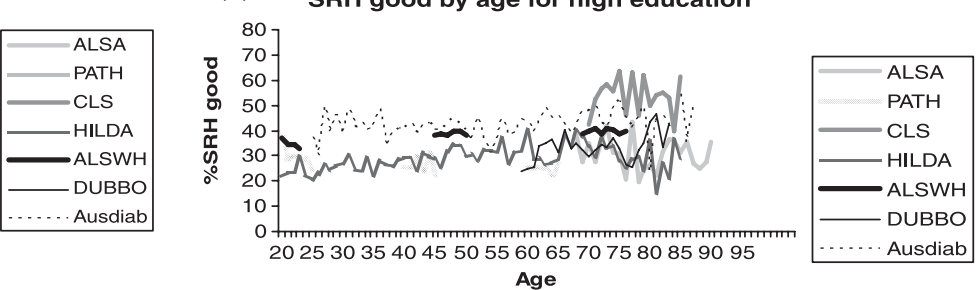

Age

(f) SRH excellent/very good by age for high education

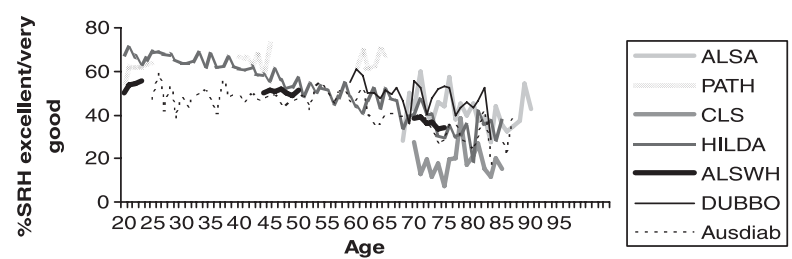


Finally, the results reported here are cross-sectional and hence reflect age differences rather than age changes in SRH. Longitudinal comparative or pooled analysis of these datasets is required to determine the extent to which the observed age patterns reflect ageing over time, as opposed to cohort effects. However, this demonstration project has shown that the compilation of results from a range of studies involving a large number of participants enables robust conclusions to be drawn about SRH at the national level. Each study included here contains valuable information on different aspects of ageing, and key outcomes that are associated with SRH. Bringing studies together in this manner offers a much stronger platform for studying health and ageing nationally, and for the development of sound evidenced-based policy.

\section{Acknowledgements}

Anstey is funded by National Health and Medical Research Council (NHMRC) Fellowship 366756. Butterworth was funded by NHMRC Public Health Fellowship 316970. This work was in part funded by NHMRC grant 410215. We thank the participants and interviewers from all studies. The ALSA has been funded by the South Australian Health Commission, the Australian Rotary Health Research Fund and by a grant from the US National Institute of Health. The National Health and Medical Research Council funded PATH, BMES, SOPS, ALSA and the Dubbo study. PATH also received a grant from the Alcohol-Related Medical Research Grant Scheme of the Australian Brewers' Foundation and a grant from the Australian Rotary Health Research Fund. The BMES was also funded by the Research and Development Grants Advisory Council. ALSWH and AUSDiab were primarily funded by the Australian Government Department of Health and Ageing with other supporters of AusDiab listed elsewhere (Dunstan 2002). The Dubbo study was also funded by the Australian Research Council. The HILDA Project was initiated by and was funded by the Commonwealth Department of Families, Community Services and Indigenous Affairs (FaCSIA) and is managed by the Melbourne Institute of Applied Economic and Social Research. The MELSHA baseline survey was funded by the Victorian Health Promotion Foundation. The findings in this paper do not reflect the views of any of the government departments or funding bodies.

\section{Key Points}

- Australian men and women report similar levels of SRH.

- SRH shows decline with increasing age, until the tenth decade.

- Low education is associated with much poorer SRH in Australia even in young adults.

- Nationally representative sampling does not necessarily result in similar results across studies, and rural studies do not necessarily provide different results from nationally representative studies.

\section{References}

1 Australian Institute of Health and Welfare (AlHW). Vision Problems in Older Australians. Canberra, ACT: AlHW, 2003.

2 Ware JE, Kosinski M, Keller SD. SF-36 Physical and Mental Health Summary Scales: A User's Manual. Boston, MA: Health Assessment Laboratory, 1994.

3 DeSalvo KB, Bloser N, Reynolds K, He J, Muntner P. Mortality prediction with a single general self-rated health question. A meta-analysis. Journal of General Internal Medicine 2006: 21: 267-275.

4 Anstey KJ, von Sanden C, Sargent-Cox K, Luszcz MA. Prevalence and risk factors for depression in a longitudinal, population-based study including individuals in the community and residential care. American Journal of Geriatric Psychiatry 2007; 15: 497-505.

5 Anstey KJ, Windsor TD, Luszcz MA, Andrews GR. Predicting driving cessation over 5 years in older adults: Psychological well-being and cognitive competence are stronger predictors than physical health Journal of the American Geriatrics Society 2006; 54: 121-126.

6 Jorm AF, Windsor TD, Dear KBG, Anstey KJ, Christensen H, Rodgers B. Age group differences in psychological distress: The role of psychosocial risk factors that vary with age. Psychological Medicine 2005; 35: 1253-1263.

7 Emmelin M, Weinehall L, Stegmayr B, Dahlgren L, Stenlund H, Wall S. Self-rated ill-health strengthens the effect of biomedical risk factors predicting stroke, especially for men - An incident case referent study. Journal of Hypertension 2003; 21: 887-896.

8 Idler EL, Russell LB, Davis D. Survival, functional limitations, and self-rated health in the NHANES I epidemiologic follow-up study, 1992. First National Health and Nutrition Examination Survey. American Journal of Epidemiology 2000: 152: 874-883.

9 Roos NP, Roos LL, Mossey J, Havens B. Using administrative data to predict important health outcomes. Entry to hospital, nursing home, and death. Medical Care 1988; 26: 221-239.

10 Blaum CS, Liang J, Liu X. The relationship of chronic diseases and health status to the health service utilisation of older Americans. Journal of American Geriatric Society 1994; 42: 1087-1093.

11 Segovia J, Bartlett RF, Edwards AC. An empirical analysis of the dimensions of health status measures. Social Science and Medicine 1989; 29: 761-768.

12 Murata C, Kondo T, Tamakoshi K, Yatsuya H, Toyoshima H. Determinants of self-rated health. Could health status explain the association between self-rated health and mortality? Archives of Gerontology and Geriatrics 2006; 43: 369-380.

13 Australian Institute of Health and Welfare (AIHW). Longitudinal studies of ageing. Canberra, ACT: AlHW, 2004. Cat. N. Age, 42.

14 Andrews G, Cheok S, Carr S. The Australian longitudinal study of ageing. Australasian Journal on Ageing 1989; 8: 31-35.

15 Dunstan DZP, Welborn T, Cameron A et al. The Australian diabetes, obesity and lifestyle study (AusDiab) methods and response rates. Diabetes Research and Clinical Practice 2002; 57: 119-129.

16 Wooden M, Freidin S, Watson N. The household, income and labour dynamics in Australia (HILDA) survey: wave 1. Australian Economic Review 2002: 35: 339-348.

17 Brown WJBL, Byles JE, Dobson AJ, Manderson L, Schofield M, Williams G. Women's health Australia: Establishment of the Australian longitudinal study on women's health. Journal of Women's Health 1996; 5: 467-472.

18 Henderson AS, Jorm AF, Mackinnon A et al. The prevalence of depressive disorders and the distribution of depressive symptoms in later life: $A$ survey using draft ICD-1- and DSM-III-R. Psychological Medicine 1993; 23: $719-729$

19 Jorm AF, Anstey KJ, Christensen H, Rodgers B. Gender differences in cognitive abilities: The mediating role of health state and health habits. Intelligence 2004; 32: 7-23.

20 Simons LA, Simons J, Powell I, Ruys J, Heller R, Lerba C. The Dubbo study: An Australian prospective community study of the health of elderly. Australia and New Zealand Journal of Medicine 1990, 783-789.

21 Broe GA, Creasey $\mathrm{H}$, Jorm AF et al. Health habits and risk of cognitive impairment and dementia in old age: A prospective study on the effects of exercise, smoking and alcohol consumption. Australian and New Zealand Journal of Public Health 1998; 22: 621-623.

22 Mitchell P, Smith W, Attebo K, Wang JJ. Prevalence of age-related maculopathy in Australia. The Blue Mountains Eye Study. Ophthalmology 1995; 102: 1450-1460.

23 Kendig H, Helme R, Teshuva K, Osborne D, Flicker L, Browning C. Health Status of Older People Project: Preliminary Findings of A Survey of the Health and Lifestyles of Older Australians. Melbourne, Vic.: Victorian Health Promotion Foundation, 1996. 